\title{
BAP31 在结直肠癌中的表达及临床意义
}

\author{
董令仪 ${ }^{\dagger}$, 姜可伟 ${ }^{\dagger}$, 张彦斌, 张辉, 禚洪庆, 崔志荣, 叶颖江 ${ }^{*}$, 王杉 \\ 北京大学人民医院胃肠外科, 外科肿瘤实验室, 北京 100044 \\ $\dagger$ 同等贡献 \\ * 联系人, E-mail: yjye101@yahoo.com.cn; dr.wangshan@yahoo.com.cn \\ 2011-02-18 收稿, 2011-05-04 接受 \\ 国家自然科学基金(30872470, 81072018)和高等学校博士学科点专项科研基金新教师类(20090001120082)资助
}

\begin{abstract}
摘要 采用组织芯片技术和免疫组织化学染色法检测 B 细胞受体相关蛋白 31 (B cell receptor associated protein 31, BAP31)在 120 例结直肠癌组织及其配对远端正常组织中的表达情况, 并 分析其与临床病理特征之间的关系. 结果显示, BAP31 主要定位于胞浆, 正常黏膜上皮呈阴性 或弱阳性表达. 在结直肠癌组织中阳性表达率为 $64.17 \%$, 明显高于正常黏膜上皮中的 $6.67 \%$, 差异具有统计学意义 $(P=0.000)$. BAP31 阴性表达与结直肠癌的低分化程度 $(P=0.001)$ 及远处转 移相关 $(P=0.036)$. BAP31 阴性患者总体生存率明显低于阳性患者 $(P=0.003)$. 因此, BAP31 可能 是判断结直肠癌预后的潜在指标, 并与肿瘤的侵袭性行为有关.
\end{abstract}

关键词

BAP31

结直肠癌

组织芯片

免疫组化
结直肠癌是世界第三大致死性疾病 ${ }^{[1]}$. 无论发展 中国家还是发达国家, 由于人口增长和老龄化程度 的加剧, 每年均有相当数目的新发病例及死亡人 数 ${ }^{[2,3]}$. 近年来, 结直肠癌的治疗取得了很大的进步, 然而由于大多数患者诊断时已经发生进展, 从而限 制了该病生存率的进一步改善 ${ }^{[4]}$. 因此, 寻找结直肠 癌早期诊断和预后标志物对于指导治疗和改善患者 的预后具有重要意义. BAP31 是本课题组前期采用蛋 白质组学技术分离鉴定得到的 1 个结直肠癌相关蛋 白, 其表达水平在结直肠癌肿瘤细胞中比正常黏膜 细胞上调超过 4 倍 ${ }^{[5]}$. 为了进一步明确其在结直肠癌 中的具体表达情况及与临床病理特征之间的关系, 我们检测了 120 例经过手术治疗结直肠癌患者中 BAP31 的表达.

BAP31 由 Kim 等人 ${ }^{[6]}$ 于 1994 年鉴定, 属于 B 细 胞受体家族蛋白的一员. 完整的 BAP31 蛋白分子可 以参与 $\mathrm{B}$ 细胞的激活, 并且是膜蛋白运输的重要介 质和细胞调亡调节子 ${ }^{[7,8]}$. BAP31 可以被裂解成在调 亡中发挥关键作用的 BAP20 蛋白片段 ${ }^{[9,10]}$. 当转人突 变的 BAP31 后, caspase-8 不能将其裂解为 BAP20, 从
而可以干扰细胞死亡相关的钙网蛋白暴露途径 ${ }^{[11]}$. 此外, 有研究显示, BAP31 在肿瘤细胞中通过依赖于 钲联蛋白结合, 可以参与内质网应激介导的细胞调 $亡^{[12]}$. BAP31 也可以作为 Bcl-2 结合蛋白, 构象激活 Bcl-2 的拮抗剂 Bax 或 Bak 蛋白, 从而促进细胞调 $亡^{[13]}$. 这些研究均表明, BAP31 在细胞调亡中发挥了 重要作用. 肿瘤细胞的显著特征之一是调亡减少, 调 亡的异常也是结直肠癌发病机制的一个主要因素 ${ }^{[14]}$. 结肠上皮细胞则对调亡高度耐受 ${ }^{[15]}$, 提示 BAP31 可 能在结直肠癌发生中发挥重要作用. 迄今, BAP31 在 结直肠癌中的表达情况尚不清楚. 因此, 本研究在较 大样本中检测了 BAP31 的表达情况及其与临床病理 特征之间的关系.

\section{1 材料与方法}

(i ) 一般资料. 连续收集北京大学人民医院胃 肠外科 2002 年 9 月 2004 年 4 月期间手术切除的结 直肠癌及配对正常黏膜组织的石蜡标本, 所有标本 均经病理形态学检查证实, 全部病例术前均未作化 疗或放疗，临床病理资料完整. 同时，收集 20 例结直 
肠腺瘤和 8 例结直肠癌肝转移灶的石蜡标本用于免 疫组化染色. 主要临床病理资料见表 1. 截止到随访 结束, 共 17 人失访, 中位随访时间为 53.3 个月 (1 78 个月), 43 人死亡, 其中 3 人死于非肿瘤性疾病(肺 炎、心肌梗死和糖尿病).

(ii) 组织芯片的制备. 复阅 HE 染色片, 挑选 上述标本中有代表性的蜡块, 对照 HE 染色片肿瘤 或远端正常组织部位, 在福尔马林固定的石蜡标本 上标注取材部位. 利用组织芯片制作仪器, 依次从 供体蜡块上穿取直径 $2 \mathrm{~mm}$ 的组织芯, 插人到受体 蜡块中, 每个病例均含有 2 个肿瘤组织芯及 2 个对 照正常组织芯. 共制备 11 张组织芯片, 每张芯片包 含 10 12 对配对的病例标本. 另外以相同方法制备 1 张包含 20 例结直肠腺瘤组织的组织芯片, 每例样 品为 1 个组织 芯, 其余 8 例肝转移灶组织则同样重 复 2 个组织芯.

(iii) 免疫组化染色. 组织芯片以 $4 \mu \mathrm{m}$ 厚度连 续切片, 采用 Envision 法进行免疫组化染色, 按照试 剂操作说明进行. 常规二甲苯脱蜡、梯度酒精水洗, 柠檬酸盐缓冲液 $(\mathrm{pH}$ 6.8) 高温修复 $15 \mathrm{~min}$, 一抗 BAP31(美国 Genetex Inc 公司)工作浓度 1:400 室温孵 育 $2 \mathrm{~h}$, 磷酸盐缓冲液代替一抗作为阴性对照.二抗 室温孵育 $30 \mathrm{~min}$, 二氨基联苯胺 (diaminobenzidine,
$\mathrm{DAB})$ 染色，常规梯度酒精脱水，二甲苯透明，中性 树脂封片.

(iv) 结果判读. 采用半定量评分系统进行结果 判读 ${ }^{[16,17]}$, 根据阳性染色面积和染色强度结果进行 综合评分. BAP31 蛋白阳性染色为细胞浆呈黄色至棕 黄色. 阳性染色面积评分为 0 , 阴性或 $<5 \% ; 1$, $5 \%$ 25\%；2，26\% 50\%；3，51\% 75\%； 4, >75\%。 染色 强度评分为 0 , 阴性; 1 , 弱阳性; 2 , 中等强度; 3 , 强 阳性. 染色综合评分为面积 $\times$ 强度, 0 分为 BAP31 蛋 白阴性表达, 1 12 分为 BAP31 蛋白阳性表达.

(V) 统计学处理. 采用 SPSS13.0 软件进行统 计学分析. 差异蛋白表达与临床病理指标之间的关 系采用卡方检验. 生存曲线分析采用 Kaplan-Meier 法, 以 Log-rank 法检测生存率的差别. $P<0.05$, 差异 有统计学意义.

\section{2 结果}

\section{1 结直肠癌患者的一般情况}

男性(74例)占 $61.67 \%$, 女性(46例)占 $38.33 \%$. 中 位年龄为 64.5 岁 (26 89 岁). 结肠癌(75 例) 占 $62.50 \%$, 直肠癌(45 例) 占 $37.50 \% .120$ 例患者的具体临床病理 特征见表 1 .

表 1 BAP31 的表达与结直肠癌患者临床病理指标之间的关系 ${ }^{\text {a) }}$

\begin{tabular}{|c|c|c|c|c|c|c|}
\hline & & \multirow{2}{*}{$n$} & \multicolumn{2}{|c|}{ BAP31 } & \multirow{2}{*}{$\chi^{2}$} & \multirow{2}{*}{$P$} \\
\hline & & & BAP31+ & BAP31- & & \\
\hline \multirow[t]{2}{*}{ 标本 } & 肿瘤组织 & & 77 & 43 & 86.728 & $0.000^{*}$ \\
\hline & 远端正常组织 & & 8 & 112 & & \\
\hline \multirow[t]{2}{*}{ 性别 } & 男性 ～～～～～ & 74 & 49 & 25 & 0.353 & 0.553 \\
\hline & 女性 & 46 & 28 & 18 & & \\
\hline \multirow[t]{2}{*}{ 肿瘤部位 } & 结肠 & 75 & 47 & 28 & 0.002 & 0.961 \\
\hline & 直肠 & 45 & 28 & 17 & & \\
\hline \multirow[t]{2}{*}{ 分化程度 } & 低分化 & 21 & 7 & 14 & 10.525 & $0.001^{*}$ \\
\hline & 中或高分化 & 99 & 70 & 29 & & \\
\hline \multirow[t]{4}{*}{ TNM 分期 } & $\mathrm{I}$ & 11 & 8 & 3 & 2.596 & 0.458 \\
\hline & II & 43 & 28 & 15 & & \\
\hline & III & 44 & 30 & 14 & & \\
\hline & IV & 22 & 11 & 11 & & \\
\hline \multirow[t]{2}{*}{ 浸润程度 } & $\mathrm{T} 1+\mathrm{T} 2$ & 19 & 15 & 4 & 2.145 & 0.143 \\
\hline & $\mathrm{T} 3+\mathrm{T} 4$ & 101 & 62 & 39 & & \\
\hline \multirow[t]{2}{*}{ 淋巴结转移 } & 无 & 55 & 38 & 18 & 0.622 & 0.430 \\
\hline & 有 & 64 & 39 & 25 & & \\
\hline \multirow[t]{2}{*}{ 远处转移 } & 无 & 96 & 66 & 30 & 4.385 & $0.036^{*}$ \\
\hline & 有 & 24 & 11 & 13 & & \\
\hline
\end{tabular}

a) 统计学分析采用卡方检验. $*, P<0.05$ 


\subsection{BAP31 的表达特征}

免疫组化结果显示, BAP31 主要分布于细胞浆, 在正常黏膜组织中染色多呈阴性或弱阳性(图 1(b), (d)和(f)), 而在肿瘤组织中表达呈不同程度的阳性 (图 1(a), (c)和(e)). 同时, 在肿瘤组织中 BAP31 表达 呈强阳性，而其相邻癌旁上皮组织中则表达阴性(图 1(h)). 在结直肠癌中, BAP31 阳性表达率为 $64.7 \%$, 明显高于在正常组织中的表达率 $6.67 \%(P=0.000)$.
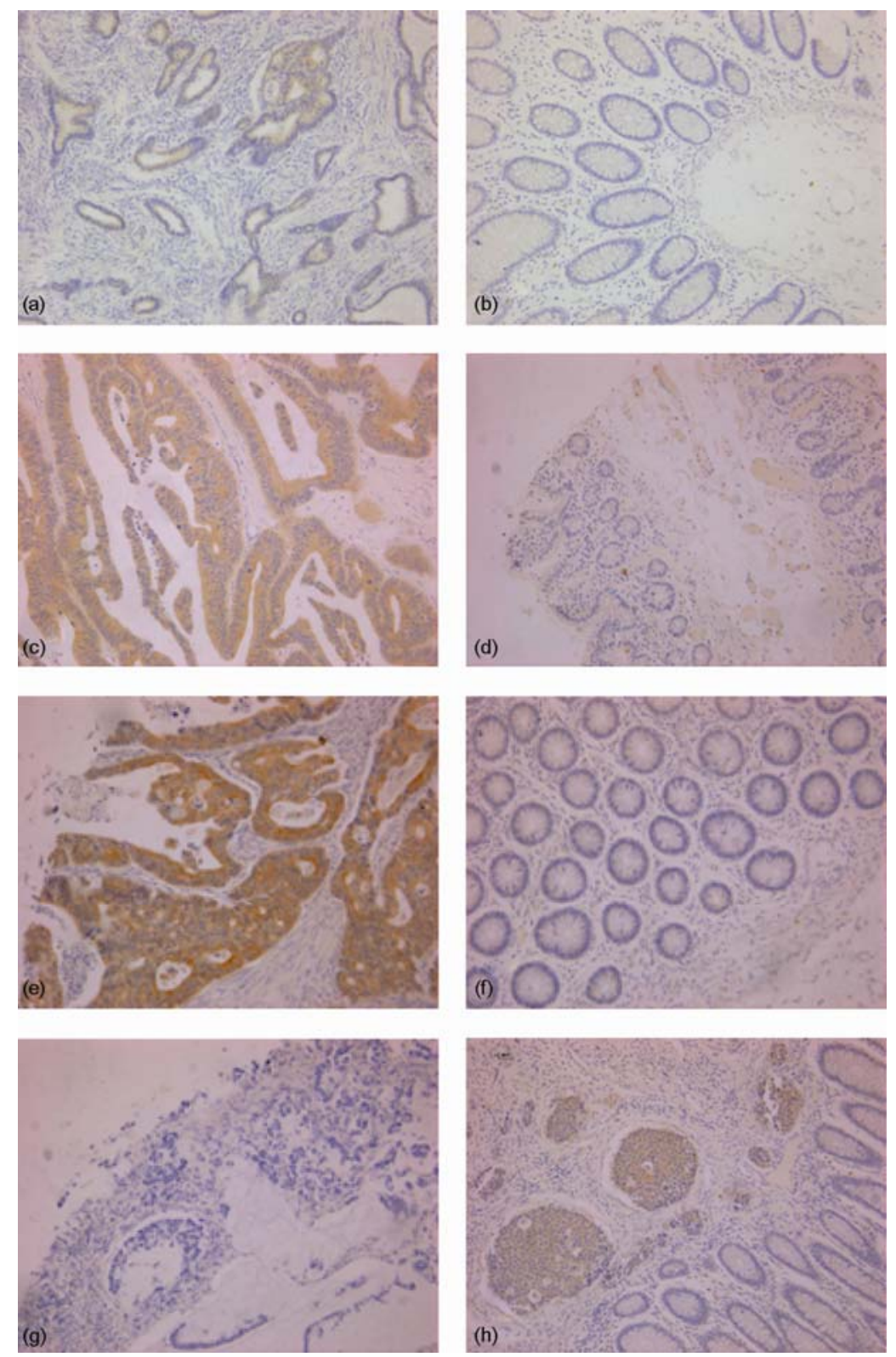

图 1 BAP31 在结直肠癌及配对正常组织中的表达

结直肠癌组织中 BAP31 ((a), (c), (e))表达阳性, 其中(a) 弱阳性表达, (c) 中度阳性表达, (e) 强阳性表达; (g) 肿瘤表达阴性; (b), (d), (f) 配对的 远端黏膜上皮组织中表达阴性或弱阳性; (h) BAP31 在肿瘤细胞表达阳性而临近的黏膜上皮细胞表达阴性. 放大倍数, $\times 100$ 

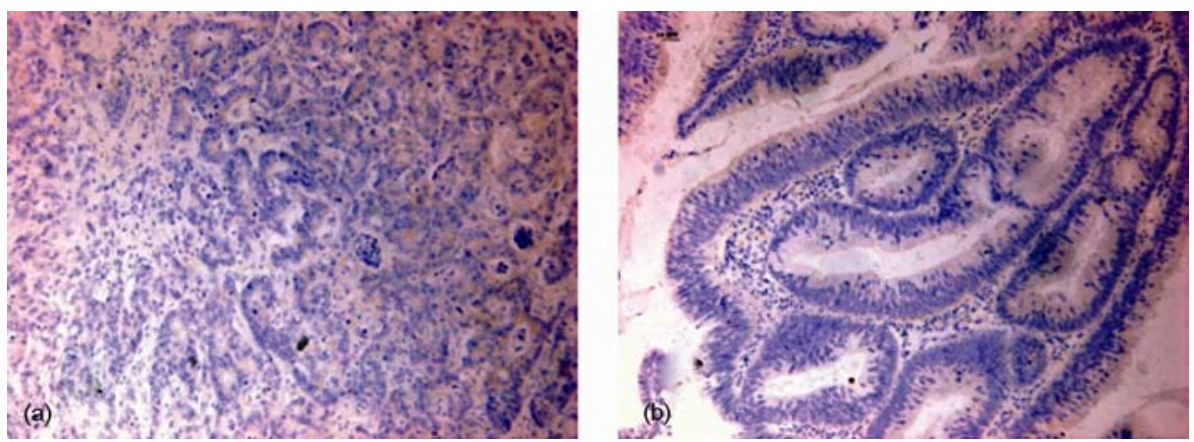

图 2 BAP31 在结直肠癌肝转移灶(a)和结肠腺瘤(b)中的表达

放大倍数, $\times 100$

表 2 BAP31 在不同结直肠肿瘤组织中的表达 ${ }^{a)}$

\begin{tabular}{|c|c|c|c|c|c|}
\hline \multirow{2}{*}{ 标本 } & \multirow{2}{*}{$n$} & \multicolumn{2}{|c|}{ BAP31 } & \multirow{2}{*}{$\chi^{2}$} & \multirow{2}{*}{$P$} \\
\hline & & BAP31+ & BAP31- & & \\
\hline 结直肠癌 & 120 & 77 & 43 & & \\
\hline 腺瘤 & 20 & 2 & 18 & 20.45 & $0.000^{*}$ \\
\hline 肝转移灶 & 8 & 1 & 7 & & $0.006^{* *}$ \\
\hline
\end{tabular}

a) *, 结直肠癌与腺瘤相比较; **, 结直肠癌与肝转移灶相比 较. 统计学分析采用四格表卡方检验或 Fisher 确切概率法, $P<0.05$, 有统计学意义

转移和 TNM (tumor node metastasis) 期以及浸润深度 无关 $(P>0.05)$. 中高分化肿瘤 BAP31 阳性表达率明显 高于低分化肿瘤 $(P=0.001)$. 无远处转移的患者 BAP31 阳性表达率明显高于有远处转移的患者 $(P=0.036)$.

\subsection{BAP31 的表达与结直肠癌预后的关系}

BAP31 阴性患者与阳性患者的总体生存率分别 为 $42.3 \%$ 和 $67.3 \%$. BAP31 阴性患者和阳性患者的平 均生存时间分别为 40.9 和 58.4 个月. 其中, BAP31 阴性患者的中位生存时间为 28 个月, 阳性患者的中 位生存时间无法评估. 生存曲线分析显示, BAP31 阳 性患者的预后好于阴性患者, 其总体生存率明显高 于阴性患者 $(P=0.003$; 图 3).

\section{3 讨论}

BAP31 是一种定位于细胞内质网膜的多面整合 蛋白, 其作为 B 细胞受体相关蛋白家族的一员, 可以 结合膜免疫球蛋白而激活 $\mathrm{B}$ 淋巴细胞 ${ }^{[18]}$. 一些研究 显示, BAP31 过表达可以增加 MHC-I 分子的细胞表 面水平 ${ }^{[19]}$, 并在细胞一般进程中发挥重要作用, 如
蛋白的早期分泌途径, 运输和调节选择性膜蛋白向 高尔基体的输出 ${ }^{[18,20]}$. 钻联蛋白通过与 BAP31 的结 合，可以调节内质网应激介导的细胞调亡 ${ }^{[12]}$. 此外, BAP31 作为 caspase-8 的优先底物, 其胞浆结构域可 以被裂解为促调亡的 BAP20 蛋白 ${ }^{[8,9,21]}$, 而在实体瘤 细胞中, caspase- 8 裂解 BAP31 形成的 BAP20 是抗肿 瘤药物依地福新(edelfosine)直接的促调亡信号 ${ }^{[22]}$.

虽然 BAP31 的 mRNA 在机体中广泛存在 ${ }^{[23]}$, 该 蛋白仅局限表达于 $\mathrm{T}, \mathrm{B}$ 淋巴细胞、胸腺、生殖细胞 和活化的腺上皮细胞中 ${ }^{[20]}$. 目前, BAP31 在肿瘤中的 具体功能尚不清楚, 而研究其与肿瘤关系的报道较 为少见. 我们首次研究了较大样本结直肠癌中 BAP31 的表达情况, 结果显示, 原发结直肠癌组织 中 BAP31 的表达较正常组织明显升高, 这与本课题 组的前期结果一致 ${ }^{[5]}$. 正常组织和腺瘤中 BAP31 的 表达多为阴性或弱阳性, 而在肿瘤中多为阳性, 并且 中高分化癌中阳性表达更多, 明显多于低分化癌. 同

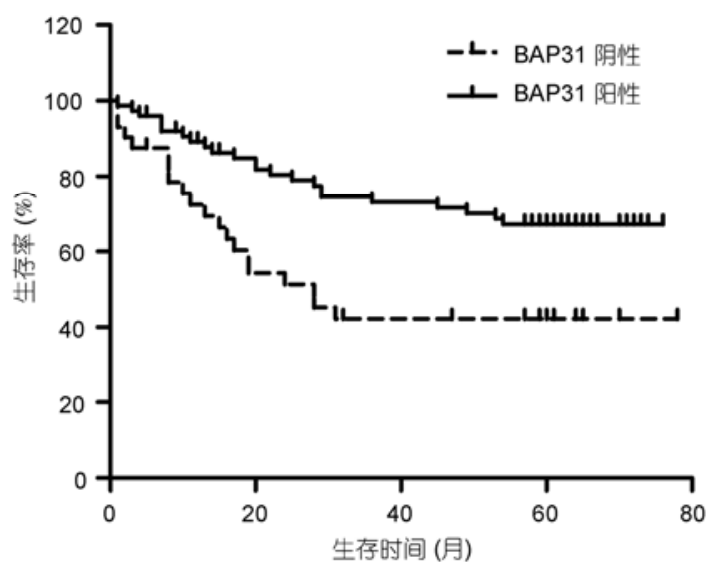

图 3 BAP31 的表达与结直肠癌患者预后的关系 BAP31 表达阴性患者的总体生存率明显低于表达阳性患者 $(P=0.003)$ 
时, BAP31 在肝转移灶中的阳性表达率较低. 这提示 BAP31 可能参与了结直肠癌的发生发展. BAP 31 的 阳性表达与肿瘤的高分化及无远处转移有关, 但是 与临床分期无关, 我们考虑可能由于 BAP31 是肿瘤 分化程度的标记物. 因此, BAP31 阳性表达的患者具 有更长的生存期. 由此推测, BAP31 可能在结直肠癌 中发挥了促调亡的作用, BAP31 裂解为促调亡蛋白 BAP20 而诱导细胞调亡，但是其作用机制尚不清楚. BAP31 表达阴性的患者生存期不仅明显短于 BAP31 表达阳性的患者, 远处转移患者的 BAP31 阴性表达
率明显低于无远处转移的患者, 因此推测 BAP31 的 上调有可能减少肿瘤的侵袭性行为, 而 BAP31 的阴 性表达与肿瘤的侵袭性行为有关.

总之, 本研究首次揭示了原发结直肠癌中 BAP31 的表达明显高于正常组织. BAP31 阴性表达 与结直肠癌的低分化、远处转移相关. BAP31 阳性患 者预后明显好于阴性患者. 由于结直肠癌患者的不 良预后，其预后因子显得异常重要. 因此, BAP31 可 能是一个潜在的结直肠癌预后标记物, 但还需要进 一步研究 BAP31 在结直肠癌中的具体功能.

\section{参考文献}

1 Jemal A, Siegel R, Ward E, et al. Cancer statistic 2008. CA Cancer J Clin, 2008, 58: 71-96

2 Winawer S J. Colorectal cancer screening. Best Pract Res Clin Gastroenterol, 2007, 21: 1031-1048

3 Jema A, Siegel R, Ward E, et al. Cancer statistics 2009. CA Cancer J Clin, 2009, 59: 225-249

4 Wolpin B, Meyerhardt J, Mamon H, et al. Adjuvant treatment of colorectal cancer. CA Cancer J Clin, 2007, 57: 168-185

5 Zhang Y, Ye Y, Shen D, et al. Identification of transgelin-2 as a biomarker of colorectal cancer by laser capture microdissection and quantitative proteome analysis. Cancer Sci, 2010, 101: 523-529

6 Kim K M, Adachi T, Nielsen P J, et al. Two new proteins preferentially associated with membrane immunoglobulin D. EMBO J, 1994, 13: 3793-3800

7 Annaert W G, Becker B, Kistner U, et al. Export of cellubrevin from the endoplasmic reticulum is controlled by BAP31. J Cell Biol, 1997, 139: $1397-1410$

8 Breckenridge D G, Stojanovic M, Marcellus R C, et al. Caspase cleavage product of BAP31 induces mitochondrial fission through endoplasmic reticulum calcium signals, enhancing cytochrome $c$ release to the cytosol. J Cell Biol, 2003, 160: 1115-1127

9 Chandra D, Choy G, Deng X, et al. Association of active caspase 8 with the mitochondrial membrane during apoptosis: Potential roles in cleaving BAP31 and caspase 3 and mediating mitochondrion-endoplasmic reticulum cross talk in etoposide-induced cell death. Mol Cell Biol, 2004, 24: 6592-6607

10 Nguyen M, Breckenridge D G, Ducret A, et al. Caspase-resistant BAP31 inhibits fas-mediated apoptotic membrane fragmentation and release of cytochrome $c$ from mitochondria. Mol Cell Biol, 2000, 20: 6731-6740

11 Madeo F, Durchschlag M, Kepp O, et al. Phylogenetic conservation of the preapoptotic calreticulin exposure pathway from yeast to mammals. Cell Cycle, 2009, 8: 639-642

12 Delom F, Emadali A, Cocolakis E, et al. Calnexin-dependent regulation of tunicamycininduced apoptosis in breast carcinoma MCF-7 cells. Cell Death Differ, 2007, 14: 586-596

13 Panaretakis T, Kepp O, Brockmeier U, et al. Mechanisms of pre-apoptotic calreticulin exposure in immunogenic cell death. EMBO J, 2009, 28: 578-590

14 Watson A J. Apoptosis and colorectal cancer. Gut, 2004, 53: 1701-1709

15 Marshman E, Ottewell P D, Potten C S, et al. Caspase activation during spontaneous and radiation-induced apoptosis in the murine intestine. J Pathol, 2001, 195: 285-292

16 Remmele W, Stegner H E. Recommendation for uniform definition of an immunoreactive score (IRS) for immunohistochemical estrogen receptor detection (ER-ICA) in breast cancer tissue. Der Pathologe, 1987, 8: 138-140

17 Magerla C, Ellinger J, Braunschweig T, et al. H3K4 dimethylation in hepatocellular carcinoma is rare compared with other hepatobiliary and gastrointestinal carcinomas and correlates with expression of the methylase Ash2 and the demethylase LSD1. Hum Pathol, 2010, 41: 181-189

18 Adachi T, Schamel W W, Kim K M, et al. The specificity of association of the IgD molecule with the accessory proteins BAP31/BAP29 lies in the IgD transmembrane sequence. EMBO J, 1996, 15: 1534-1541

19 Ladasky J J, Boyle S, Seth M, et al. Bap31 enhances the endoplasmic reticulum export and quality control of human class I MHC molecules. J Immunol, 2006, 177: 6172-6181 
20 Manley H A, Lennon V A. Endoplasmic reticulum membrane-sorting protein of lymphocytes (BAP31) is highly expressed in neurons and discrete endocrine cells. J Histochem Cytochem, 2001, 49: 1235-1244

21 Wang B, Nguyen M, Breckenridge D G, et al. Uncleaved BAP31 in association with A4 protein at the endoplasmic reticulum is an inhibitor of Fas-initiated release of cytochromec from mitochondria. J Biol Chem, 2003, 278: 14461-14468

22 Nieto-Miguel T, Fonteriz R I, Vay L, et al. Endoplasmic reticulum stress in the proapoptotic action of edelfosine in solid tumor cells. Cancer Res, 2007, 67: 10368-10378

23 Mosser J, Sarde C O, Vicaire S, et al. A new human gene (DXS1357E) with ubiquitous expression, located in Xq28 adjacent to the adrenoleukodystrophy gene. Genomics, 1994, 22: 469-471

·动 态 .

\section{《照亮地球: 过去、现在与未来》一 IODP(2013 2023)新科学计划发布}

2011 年 6 月 16 日, 国际综合大洋钻探计划(Integrated Ocean Drilling Program, 简称 IODP)在荷兰皇家艺术与科学 学院召开新闻发布会, 正式公布 2013 年 10 月以后 10 年的 新科学计划—《照亮地球: 过去、现在与未来(Illuminating Earth's Past, Present, and Future)》(2013 2023). 2013 年 10 月开始的 IODP 将更名为国际大洋发现计划(International Ocean Discovery Program), 英文简称仍为 IODP.

目前正在执行的 IODP 计划(2003 2013)是承接“大洋 钻探计划 (ODP, 1985 2003)” 和 “深海钻探计划 (DSDP, $1968 \sim 1983)$ ”, 于 2003 年 10 月正式启动的. 其科学目标以 “地球系统科学”思想为指导, 探索地球深部与表层系统 的连接, 计划打穿大洋壳, 揭示地震机理, 查明深部生物 圈和天然气水合物, 理解极端气候和快速气候变化的过程, 同时为深海新资源勘探开发、环境预测和防震减灾等服务. 自 2009 年以来, IODP 积极筹备 2013 年以后的新发展阶段, 包括规划新科学计划、改组科学咨询结构、探索运行新模 式等. 其中, 这次发布的 2013 2023 年新科学计划经过了 国际学术界多次会议讨论, 组织权威专家编写; 其初稿在 2010 年 8 月已向社会公布, 多方面征求科学界意见, 修改 后再送资深科学家评审, 最终于 2011 年 6 月定稿. 这本共 84 页的新科学计划共包括 4 个方面科学主题:

(1) 气候和海洋变化: 解读过去, 预示未来. 海底的沉 积物岩芯提供了过去气候变化的记录, 可以帮助在空间和
时间尺度上更好理解地球系统过程. 过去的记录可以帮助 检验将来气候变化预测的模型.

(2) 生物圈前沿: 深部生命及生物演化的环境驱动. 科学大洋钻探对于研究海洋和陆地上的生命演化非常重要, 深部生物圈对于地球的环境和生命演化的作用还不清楚.

(3) 地球的连接: 深部过程及其对表层环境的影响. 洋 壳、俯冲带、构造成因的沉积和火山地层蕴含了深部地球动 力过程的记录, 这一过程控制了地球表面的形态和环境.

(4) 地球在运动: 人类时间尺度上的过程与灾害. 原 位、现场的观测实验对于理解很多短时间尺度的地球系统 过程来说至关重要.

中国自 1998 年加人 ODP 和 IODP 以来一直是国际大 洋钻探领域的积极成员, 于 1999 年春在南海实现了中国 海域的首个大洋钻探航次—ODP 第 184 航次, 该航次由 中国科学家设计和主持, 使我国海洋地质学研究一举进人 国际深海研究的前沿. 2013 年以后, 中国将加强参与 IODP 的力度, IODP 这本 2013 2023 年新科学计划涵盖了地球科 学的诸多领域, 这为我国科学家投身于海洋科学钻探研究 提供了更多机会. IODP 新科学计划全文可在中国 IODP 网站 (http://www.iodp-china.org/)下载, 供感兴趣的科学家参考.

《科学通报》编辑部 